\title{
Discrete and continuum modelling of soil cutting
}

\author{
C. J. Coetzee
}

Received: 7 November 2013 / Revised: 20 January 2014 / Accepted: 20 April 2014 / Published online: 29 April 2014

(C) Springer International Publishing Switzerland 2014

\begin{abstract}
Both continuum and discrete methods are used to investigate the soil cutting process. The Discrete Element Method (DEM) is used for the discrete modelling and the Material-Point Method (MPM) is used for continuum modelling. MPMis a so-called particle method or meshless finite element method. Standard finite element methods have difficulty in modelling the entire cutting process due to large displacements and deformation of the mesh. The use of meshless methods overcomes this problem. MPM can model large deformations, frictional contact at the soil-tool interface, and dynamic effects (inertia forces). In granular materials the discreteness of the system is often important and rotational degrees of freedom are active, which might require enhanced theoretical approaches like polar continua. In polar continuum theories, the material points are considered to possess orientations. A material point has three degrees-of-freedom for rigid rotations, in addition to the three classic translational degrees-of-freedom. The Cosserat continuum is the most transparent and straightforward extension of the nonpolar (classic) continuum. Two-dimensional DEM and MPM (polar and nonpolar) simulations of the cutting problem are compared to experiments. The drag force and flow patterns are compared using cohesionless corn grains as material. The corn macro (continuum) and micro (DEM) properties were obtained from shear and oedometer tests. Results show that the dilatancy angle plays a significant role in the flow of material but has less of an influence on the draft force. Nonpolar MPM is the most accurate in predicting blade forces, blade-soil interface stresses and the position and orientation of shear bands. Polar MPM fails in predicting the orientation of the shear band, but is less sensitive to mesh size and mesh ori-
\end{abstract}

C. J. Coetzee $(\bowtie)$

Department of Mechanical and Mechatronic Engineering, University of Stellenbosch, Private Bag X1, Matieland 7602, South Africa

e-mail: ccoetzee@sun.ac.za entation compared to nonpolar MPM. DEM simulations show less material dilation than observed during experiments.

Keywords Discrete element method - Material-point method - Cosserat continuum · Flat blade Passive problem . Soil cutting

\section{Introduction}

Earthmoving equipment is not only used for mining, it also plays an important role in the agricultural and earthmoving industries. The equipment is highly diverse in shape and function, but most of the soil cutting machines can be categorised into one of three principal classes: blades, rippers and buckets or shovels. The basic shape of earthmoving tools has not changed a great deal since antiquity, although most are operated today by mechanical power sources and their construction benefits from modern metallurgical engineering.

The design of effective and efficient implements begins with the analysis of the soil failure in order to predict the forces and energy required by the implements. The simplest form of soil cutting or tillage is that of a flat blade moving through the soil. Karmakar [1] summarises the different approaches used to model soil cutting. Six major methods can be identified, namely empirical/semi-empirical, dimensional analysis, Finite Element Methods (FEM), Discrete Element Methods (DEM), Artificial Neural Networks (ANN) and Computational Fluid Dynamics (CFD).

\subsection{Analytical methods}

Empirical and semi-empirical analytical models are based on the physics of soil, tool configuration and simplifying assumptions. The methods of Coulomb [2] and Perumpral 
$[3,4]$ assume that the material in front of the blade fails and moves as a rigid body. The sum of all forces acting on this rigid body can be used to solve for the forces acting on the blade. Sokolovski [5] used a totally different method based on the theory of plasticity. This theory is based on the assumption that a state of failure exists at any point within a certain area (zone ruptures) or on a certain curve (line ruptures). By means of this assumption and using the equations of equilibrium, it is possible to solve soil pressure problems. The material is assumed to be macroscopically homogeneous and in a critical state. The definition for critical state is when the material or part thereof is stressed to the limit of the Mohr-Coulomb yield criterion at the point just before slip occurs. The analysis is conducted for static equilibrium and no dynamic effects are taken into account. Deformation effects such as dilation are not accounted for. A set of differential equations, derived from the Mohr-Coulomb yield criterion and the equations of equilibrium, is numerically solved using the method of characteristics and appropriate boundary conditions and material properties.

The analytical methods discussed here, are based on passive earth pressure theory and assumptions of a preliminary soil failure pattern [1]. Dynamic effects, complicated tool geometry and large deformations can not be modelled using these methods. Discrete element methods and finite element methods can be used to overcome some of these problems.

\subsection{Discrete element methods}

The discrete element methods are based on the simulation of the motion of granular material as separate particles. DEM was first applied to soils by Cundall and Strack [6]. Calculations performed during a DEM simulation alternate between the application of Newton's second law to the particles and a force-displacement law at the contacts. DEM has the advantage that it can easily be used for the simulation of granular flow subjected to large deformations and free boundaries. The main problem with DEM is how to specify the micro-properties (particle contact properties) so that the flow on macro-level of thousands of particles behaves in the same way as real granular flow. Laboratory experiments [7] (e.g. shear tests, biaxial tests and oedometer tests) or in-situ tests [8] are necessary to determine these properties before any useful modelling and predictions can be made. Bohatier and Nouguier [9] modelled a flat blade cutting through a granular material using DEM. The effect of tool velocity on the draft force was shown, but no experimental results were presented.

\subsection{Finite element and meshless methods}

The entire cutting process involves large displacements of the blade and large deformation of the material being cut, which makes it difficult to model with classic finite element methods due to the distortion of the mesh. As a remedy, remeshing techniques [10] may be used, but all the state variables have to be mapped from the distorted mesh to a newly-defined one. Such a mapping introduces additional computational errors and makes them ineffective [11]. In the work presented here, a so-called meshless finite element method is used to overcome this problem.

Although meshless methods originated about twenty years ago, little research effort has been devoted to them until recently. The Material-Point Method (MPM, also called Particle-in-Cell, PIC) is a so-called particle meshless method. Particle methods can be characterised as methods where the solution variables are attributed to Lagrangian point masses instead of computational cells [12].

Sulsky et al. [13] developed a MPM method applicable to solid mechanics that can be used to model dynamic impact, penetration and large deformations. MPM uses two discretisations of the material, one based on a computational mesh and the other based on a collection of material points or 'particles'. This approach combines the advantages of Eulerian and Largrangian descriptions of the material while avoiding the shortcomings of each. The equations of motion are solved in a Lagrangian frame on a computational grid, using standard finite element methods. Convection is modelled by moving the material points in the computed velocity field. Each material point carries its material properties without error while it is moved. Since all the state variables are assigned to the numerical material points, the information carried by these points is enough to characterise the flow and the grid carries no permanent information. Thus, the grid can be discarded and reconstructed for computational convenience at each time step. The MPM formulation results in an automatic no-slip condition between different bodies. Bardenhagen et al. [14] developed an algorithm that precludes interpenetration of different bodies, but allows frictional slip at the contacting boundary nodes. This contact model is used to model the frictional soil-blade interface. See "Appendix" for a more detailed description of MPM.

\subsection{Polar continuum}

In granular materials the discreteness of the system is often important and rotational degrees of freedom are active, which might require enhanced theoretical approaches like polar continua [15]. The concept of polar continua naturally brings a length scale into the continuum theory.

In polar continuum theories, the material points are considered to possess orientations. A material point has three degrees-of-freedom for rigid rotations, in addition to the three classic translational degrees-of-freedom. Eringen [16] describes polar continua in detail. The Cosserat continuum is the most transparent and straightforward extension of classic or nonpolar continuum models and was proposed by $\mathrm{E}$. 
and F. Cosserat in 1909 [17]. The nonpolar constitutive laws must be adapted to include the rotational degrees-of-freedom which leads to new laws such as polar elasticity and polar plasticity. The rotations are induced by couple stresses within the continuum. The presence of couple stresses result in a stress tensor which is no longer symmetric, as in the case of a nonpolar continuum.

In this paper, soil cutting is modelled using DEM, nonpolar continuum MPM, and polar continuum MPM. The predicted blade draft force and material flow behaviour are compared to experimental results [18-20]. The experiments were performed under conditions that resulted in mainly two-dimensional flow of the material. The main reason for this is that the flow patterns could be accurately visualised and compared to the results from two-dimensional numerical approaches. Although the flow of material around earthmoving and agricultural equipment would be three-dimensional, this two-dimensional approach provides more insight of the material flow directly in front of the cutting tool.

During the initial stages of cutting, shear bands develop in front of the blade and during the later stages the material experiences large deformations and free surface flow. The ability of DEM and MPM to model these phenomena is investigated.

\section{Experimental and numerical setup}

Two-dimensional experiments were performed by moving a vertical flat blade ( $350 \mathrm{~mm}$ high) between two glass panels ( $200 \mathrm{~mm}$ apart). The forces acting on the blade could be measured and the flow patterns could be observed. The blade was given a constant horizontal velocity of $10 \mathrm{~mm} \mathrm{~s}^{-1}$. For a detailed description of the experimental setup and data logging see [18] and [20].

Rowlands [21] observed that seed grains are suitable for experimental testing and closely resemble natural granular flow into dragline buckets. The grains have a relatively low friction coefficient with glass, which makes it a good material for experiments. The seed grains were also found suitable for DEM simulations because the stiffness of the grains is less than the stiffness of, say, gravel. The smaller stiffness results in a larger time step and decreases the total computing time [18]. For a close-packed assembly the stable time step is roughly inversely proportional to the particle stiffness [22]. Coetzee and Els [20] used direct shear and oedometer tests to determine the corn micro (particle) properties to be used during the DEM simulations.

A Mohr-Coulomb constitutive model (perfect-plastic) [23] was used for nonpolar modelling and a polar DruckerPrager model (perfect-plastic) [24] for polar modelling. Mouazen and Neményi [25] has shown that perfect-plasticity is a valid assumption for FEM modelling of soil tillage. Plane strain conditions were assumed [26].
Table 1 Material properties

\begin{tabular}{lll}
\hline Continuum macro properties & Symbol & Corn \\
\hline Young's modulus & $E$ & $2.76 \mathrm{MPa}$ \\
Poisson's ratio & $v$ & 0.2 \\
Density & $\rho$ & $778 \mathrm{~kg} \mathrm{~m}^{-3}$ \\
Friction angle & $\phi$ & $26^{\circ}$ \\
Dilatancy angle & $\psi$ & $2^{\circ}, 10^{\circ}, 26^{\circ}$ \\
Cohesion & $c$ & $0 \mathrm{~Pa}$ \\
Cosserat shear modulus & $G^{c}=2 G$ & $2.3 \mathrm{MPa}$ \\
Characteristic length & $l$ & $10 \mathrm{~mm}$ \\
Moment of inertia & $J_{33}=0.5(0.5 l)^{2}$ & $12.5 \times 10^{-6} \mathrm{~m}^{2}$ \\
Friction with steel & $\phi_{s}$ & $14^{\circ}$ \\
\hline DEM micro properties & $S_{y m b o l}$ & $\mathrm{Corn}^{2}$ \\
\hline Particle stiffness & $k_{n}=k_{s}$ & $420 \mathrm{kN} \mathrm{m}^{-1}$ \\
Particle density & $\rho_{p}$ & $855 \mathrm{~kg} \mathrm{~m}^{-3}$ \\
Particle friction & $\mu$ & 0.1 \\
\hline
\end{tabular}

The corn grains had an average size of more or less $10 \mathrm{~mm}$ [20]. The characteristic length used in the polar continuum, assumed this value [24]. The moment of inertia of each grain was calculated by assuming a cylinder with a diameter equal to the internal length. The other material properties are summarised in Table 1. For a detailed description of the DEM calibration and model, see [20]. The material dilatancy angle could not be determined from the shear test and oedometer test data $[18,20]$. A reverse calibration procedure, described as part of the results, was used to determine the dilatancy angle. The blade was modelled as a rigid body.

In the DEM model, the initial conditions were generated by letting material (approximately 10,000 particles) fall from a fixed height along the total length of the test rig [18,20]. For MPM modelling, the bottom boundary was fixed in the horizontal, vertical and rotational (polar only) directions and the two side boundaries in the horizontal and rotational (polar only) directions. The boundaries were far away from the blade and had no significant effect on the results. No polar rotation was allowed at the blade-material interface [24]. The material stresses were initialised using a coefficient of earth pressure $K_{o}=1-\sin \phi$ [28].

Commercial DEM software, $P F C^{2 D}$ [22], was used and the MPM code was developed at Stellenbosch University in collaboration with the University of Stuttgart [19].

\section{Results}

\subsection{Material flow}

In the first part of the analysis, the material flow is investigated by mainly looking at the ability of the different numer- 
Fig. 1 Comparison between experimental [20], DEM [20] and nonpolar MPM results of corn flowing in front of the blade. Displacement $=100 \mathrm{~mm}$ and $200 \mathrm{~mm}$

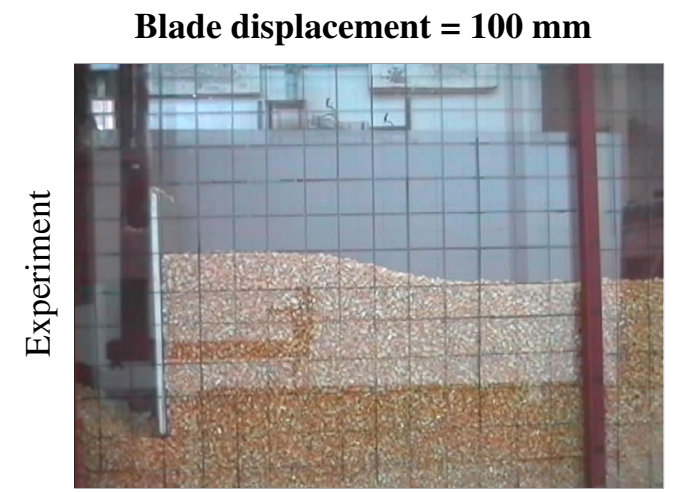

Blade displacement $=200 \mathrm{~mm}$
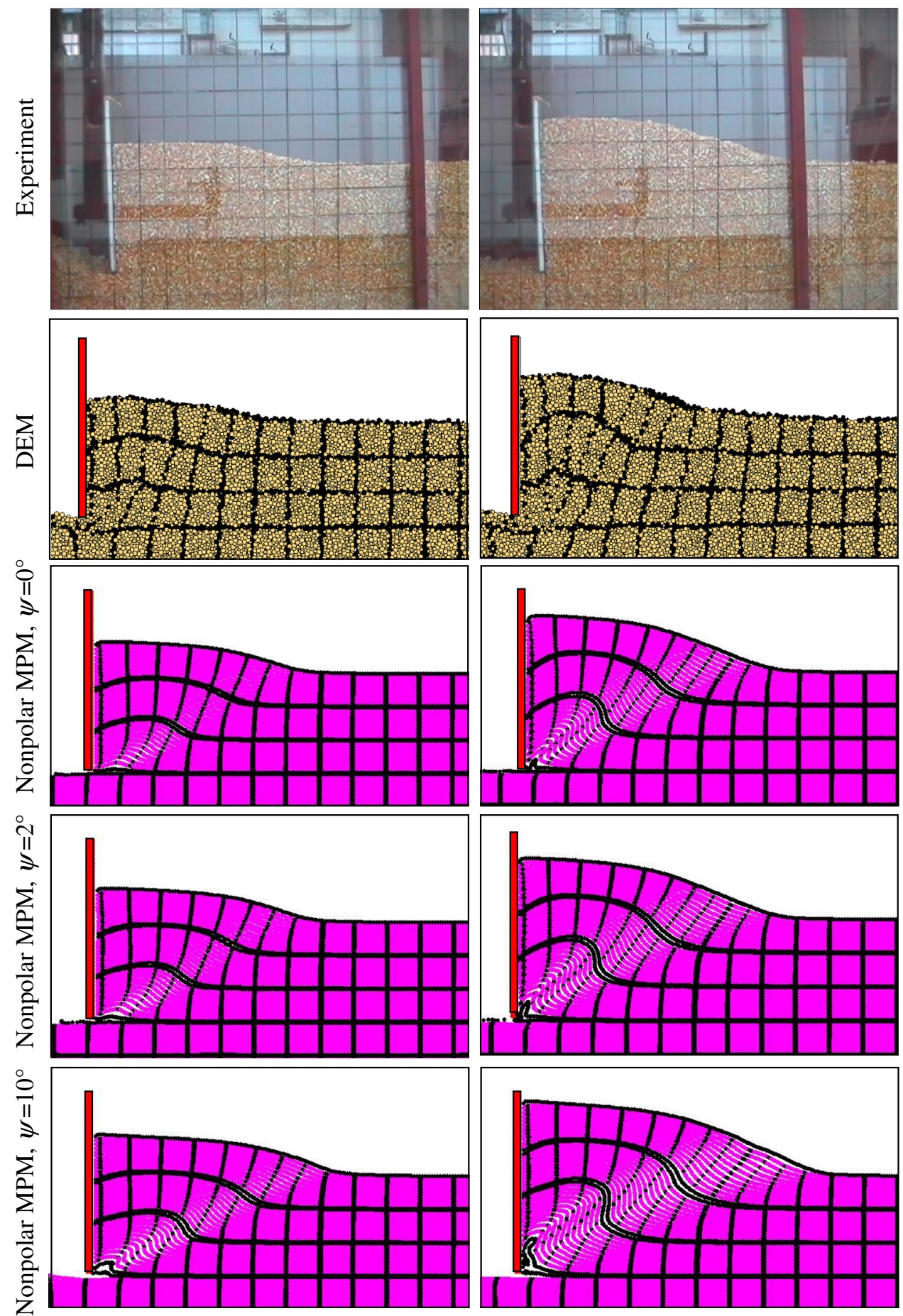

ical methods to predict the free surface. Figures 1 and 2 show the observed flow of material in front of the blade and the results from DEM and nonpolar MPM simulations (the experimental and DEM results are from [20]). The initial blade depth was $h=200 \mathrm{~mm}$. With only the dilatancy angle unknown, the simulation was repeated for three different values $\psi=2^{\circ}, \psi=10^{\circ}$ (non-associated) and $\psi=\phi=26^{\circ}$ (associated flow rule). There is good qualitative agreement between the DEM and MPM flow patterns, as shown by the coloured layers.

Curves were fitted to the free surfaces to make a quantitative comparison of the flow of material, Fig. 3. The free surfaces exhibit the effect of the dilatancy angle: with an increase in dilatancy angle, there is an increase in material 
Fig. 2 Comparison between experimental [20], DEM [20] and nonpolar MPM results of corn flowing in front of the blade. Displacement $=300 \mathrm{~mm}$ and $400 \mathrm{~mm}$

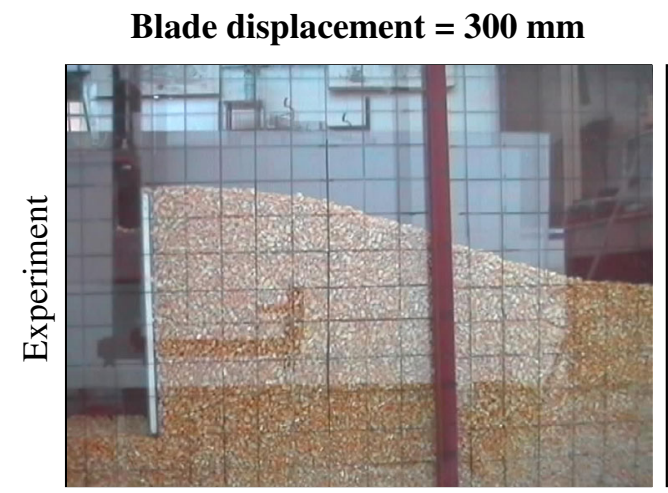

Blade displacement $=400 \mathrm{~mm}$
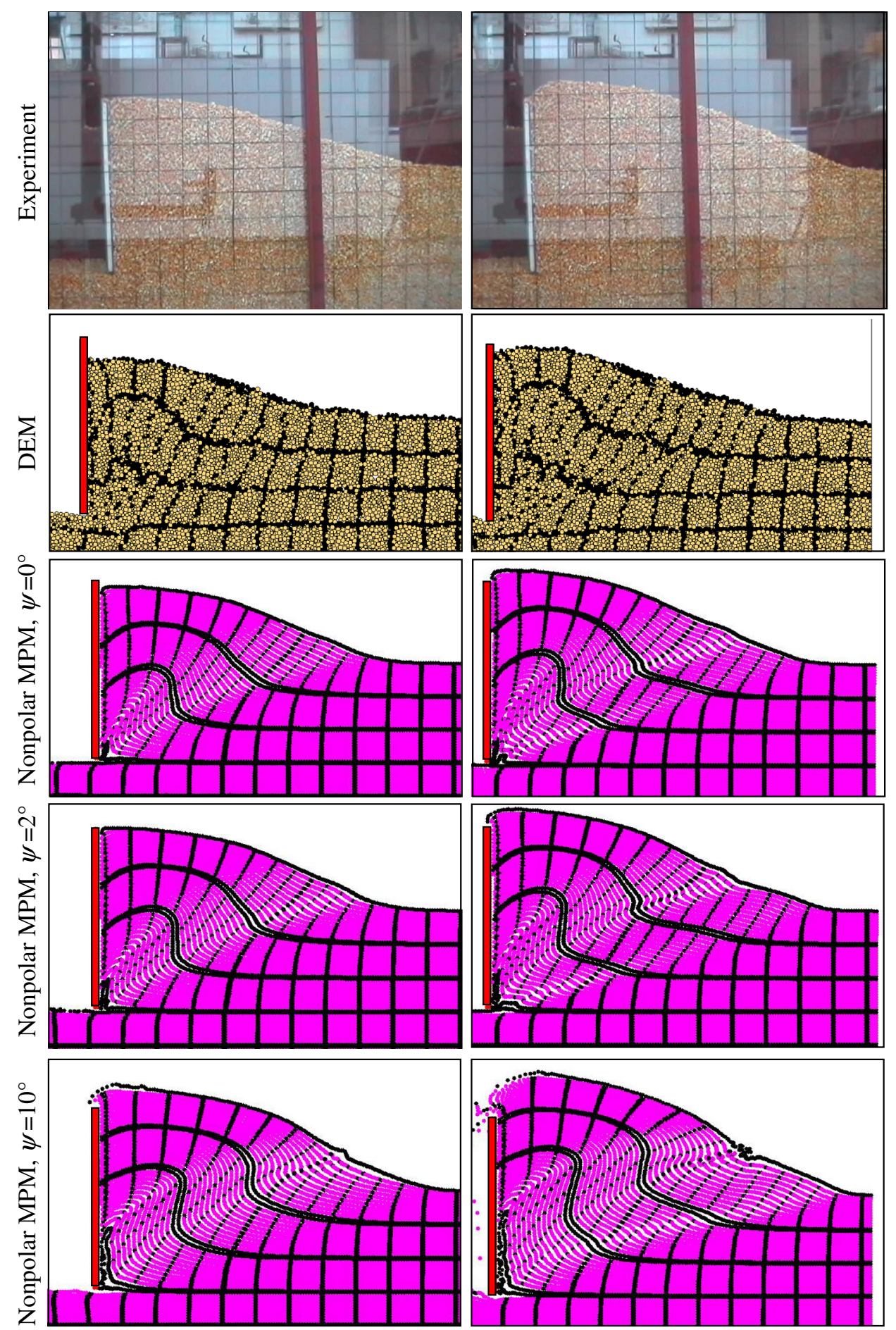

volumetric expansion. The free surface is the most accurately predicted using a dilatancy angle $\psi=2^{\circ}$ and this value is assumed for all further simulations.

The MPM simulation was repeated with a Cosserat (polar) continuum. The friction- and dilatancy parameters are calculated as proposed by Tejchman [24]. A comparison of the polar and nonpolar MPM flow patterns is given in Fig. 4. At a displacement of $100 \mathrm{~mm}$ there is good agreement, but as the blade moves further, the polar continuum seems to show less dilation than the nonpolar continuum. The curves fitted to the free surfaces are shown in Fig. 5. From these curves it can be seen that nonpolar MPM predicts the free surface the most accurately. The DEM free surface is in close agreement with the polar MPM free surface up to a displacement of 


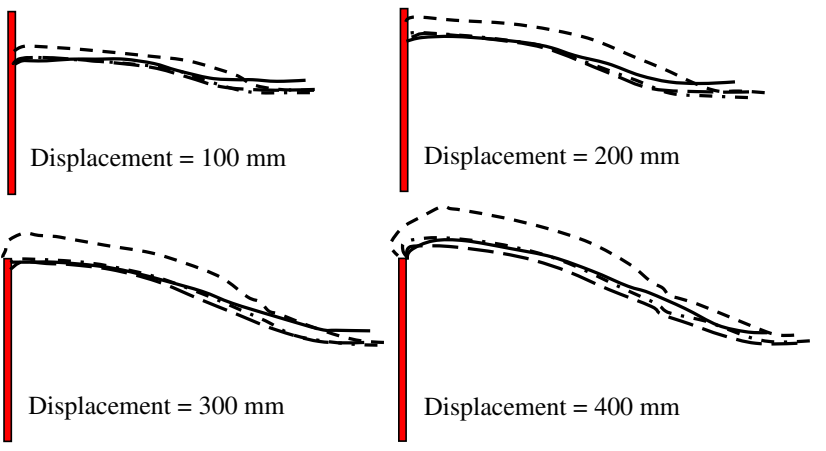

\begin{tabular}{ll} 
Experiment & $-\ldots-$ Nonpolar MPM $\psi=0^{\circ}$ \\
\hdashline$-\cdots-\cdots$ Nonpolar MPM $\psi=10^{\circ}$ & $-\cdots-\cdots$ Nonpolar MPM $\psi=2^{\circ}$
\end{tabular}

Fig. 3 Comparison of the free surfaces using different dilatancy angles $\psi$ and nonpolar MPM

Fig. 4 Comparison between polar and nonpolar MPM results of corn flowing in front of the blade with $\psi=2^{\circ}$
$200 \mathrm{~mm}$, but both fail to predict the experimental free surface accurately.

The forces that should be applied to the blade to move it at constant velocity are shown in Fig. 6 for different values of $\psi$. The force in the vertical direction is negative, which indicates that the material tends to push the blade upwards, hence a negative force should be applied to the blade to prevent vertical movement. The draft force (horizontal direction) is defined as the force needed to push or pull a blade through the material, in the direction of the motion and is important in industrial applications such as ploughs, tillers and bulldozers. Over the first part of the displacement the responses, using nonpolar MPM, are the same because the material is in a total elastic state. In the plastic regions, how-

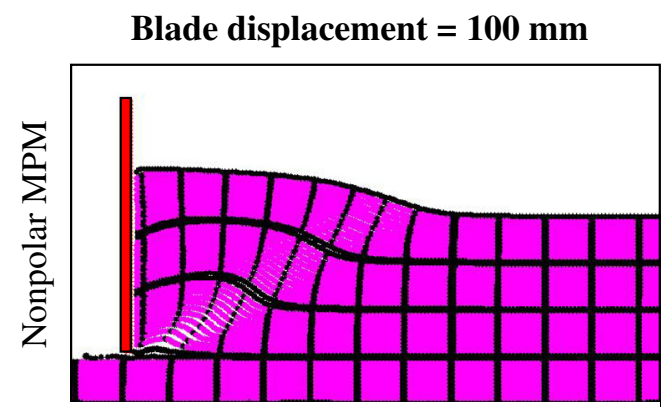

Blade displacement $=200 \mathbf{m m}$
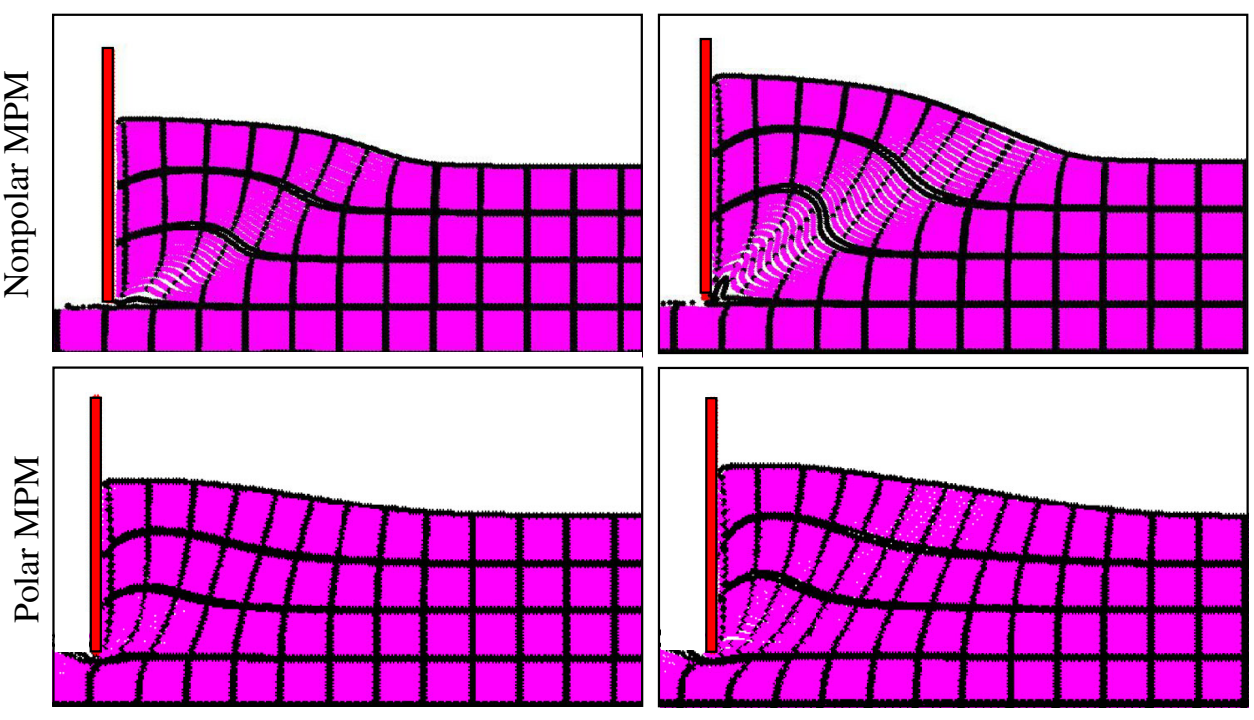

Blade displacement $=400 \mathrm{~mm}$
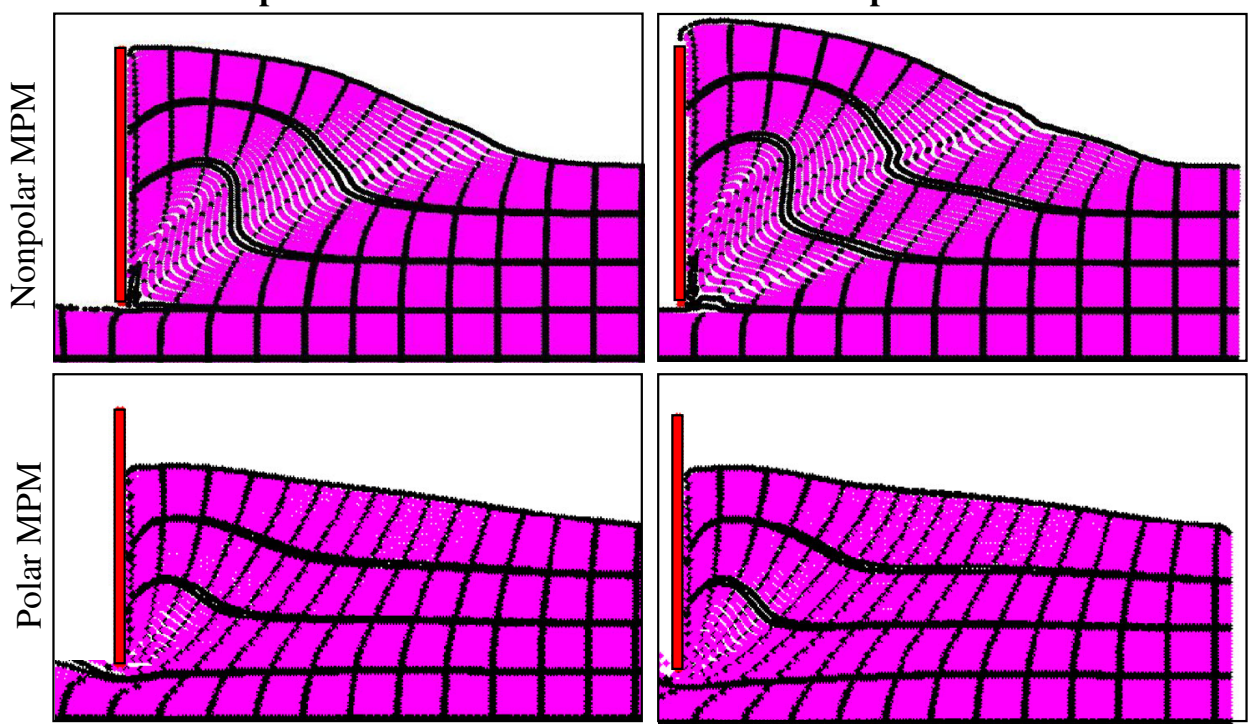
ever, there is an increase in blade forces with an increase in dilatancy angle. This phenomenon has also been observed for cone penetration tests [27]. The polar MPM result, with $\psi=2^{\circ}$ is also shown. Polar MPM, however, predicts forces $13 \%$ lower than nonpolar MPM with $\psi=2^{\circ}$. Polar MPM also shows an initial elastic stiffness that slightly differs from that of nonpolar MPM. According to Tejchman [24], the Cosserat shear modulus $G^{c}$ has an influence on the material stiffness.

\subsection{Blade forces}

In the second part of the analysis, the ability of the different numerical models to predict the blade forces is investigated. As shown in Fig. 6, the blade forces stabilizes after $10 \mathrm{~mm}$ of displacement. During these initial stages, there is little upheave (bulldozing) of the material in front of the blade and the forces are mainly due to material failure. Only if the blade is moved further $(>60 \mathrm{~mm})$ does the upheave of the material tend to have the effect of increasing the blade forces. In order to make comparisons, only blade forces up to a displacement of $35 \mathrm{~mm}$ are considered, i.e., the effect of upheaving does not play a role.

Using the dilatancy angle $\psi=2^{\circ}$ and nonpolar MPM, the effect of blade depth $h$ on the blade forces is shown in Fig. (7), using depths $h=150,250$ and $350 \mathrm{~mm}$. As expected, the forces increase with an increase in blade depth. In order to compare the MPM results to that from DEM, experiments and Sokolovski's method of characteristics [5], the average draft forces are calculated. The average draft forces were calculated from Fig. 7, using the data between 15 and $35 \mathrm{~mm}$ in displacement. A similar procedure was applied to the experimental results, DEM results, and polar MPM results. All the results are shown in Fig. 8 and show the same general trend that the draft force is exponentially dependent on the blade depth. The nonpolar MPM results are in close agreement with the results from Sokolovski's method, overestimating it by 12,6 and $5 \%$ for the depths $h=150,250$ and $350 \mathrm{~mm}$ respectively. The nonpolar MPM results underestimate the experimental results by 25,11 and $9 \%$ for the depths $h=150,250$ and $350 \mathrm{~mm}$ respectively. There might be different reasons for the fact that the simulations predict lower draft forces than were measured. Although the friction coefficient between the corn and glass is low, there are still friction forces which will result in measured values that are higher than it would have been under purely plane strain two dimensional conditions. Polar MPM predicts draft forces 30,21 and $20 \%$ lower than the measured values for the three different depths respectively and 28, 14 and $10 \%$ lower than the nonpolar MPM results. Tejchman [24] reports a $30 \%$ difference in polar and nonpolar continuum results in modelling a strip footing with standard FEM. The percentage difference in polar and nonpo-

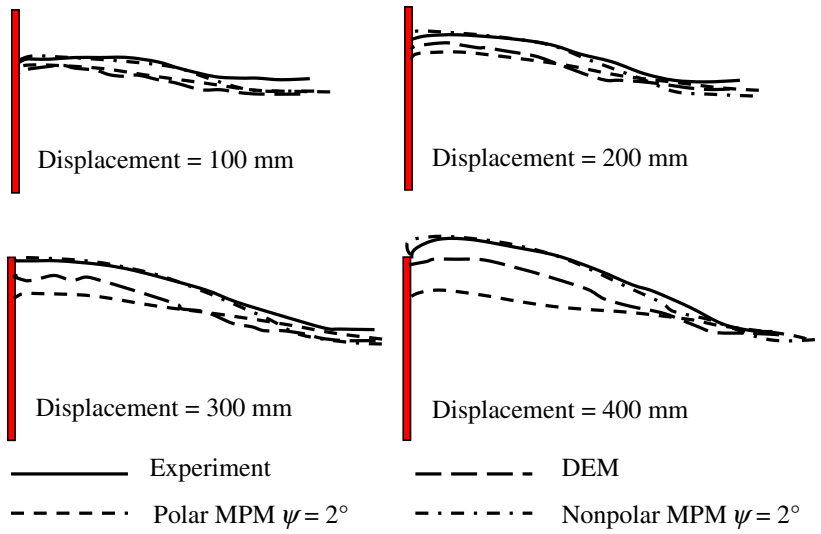

Fig. 5 Comparison of the experimental, $\operatorname{polar} \operatorname{MPM}\left(\psi=2^{\circ}\right)$, nonpolar $\operatorname{MPM}\left(\psi=2^{\circ}\right)$ and DEM free surfaces

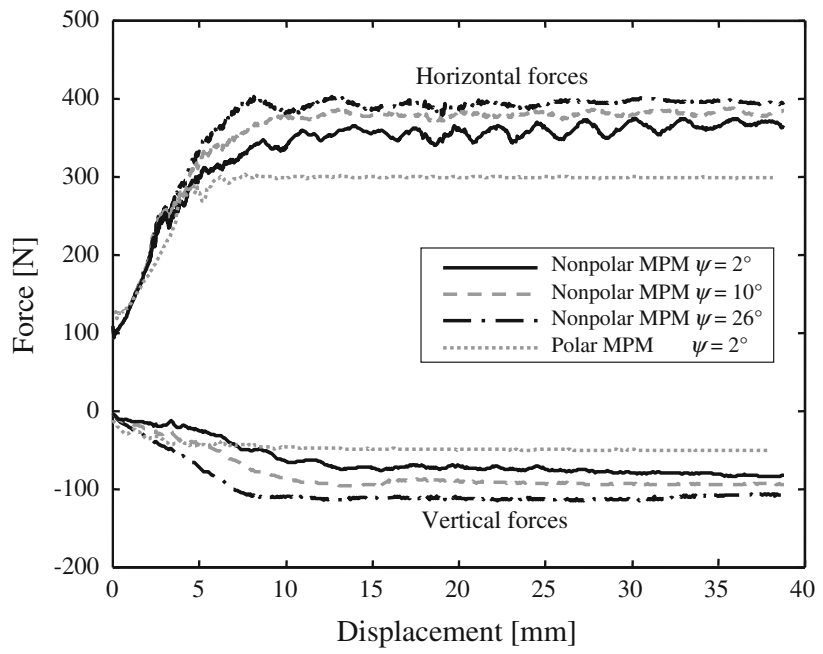

Fig. 6 The effect of the dilatancy angle $\psi$ on the blade forces with blade depth $h=350 \mathrm{~mm}$

lar MPM results compared to experiments seems to decrease with an increase in blade depth, i.e. an increase in stress. The DEM result, compared to the measured values, predicts a higher draft force with $h=150 \mathrm{~mm}$ and $h=250 \mathrm{~mm}$ and a lower draft force with $h=350 \mathrm{~mm}$.

Figure 9 shows the normal and shear stress at the blade as predicted by nonpolar MPM, polar MPM and Sokolovski's method. The continuum results were obtained from the nodal forces acting on the blade. The irregularity in the stress close to the bottom edge of the blade is due to the unit normal vector at the bottom node which is not horizontal and possible stress concentrations. The nonpolar MPM results are in good agreement with the Sokolovski predictions. The polar MPM results are shown using two boundary conditions: no rotation at the blade $w^{c}=0$ and free rotation at the blade. Polar MPM predict normal and shear stresses lower than that of nonpolar MPM and Sokolovski's method. Having no rotation or free rotation at the blade has little effect on the nor- 


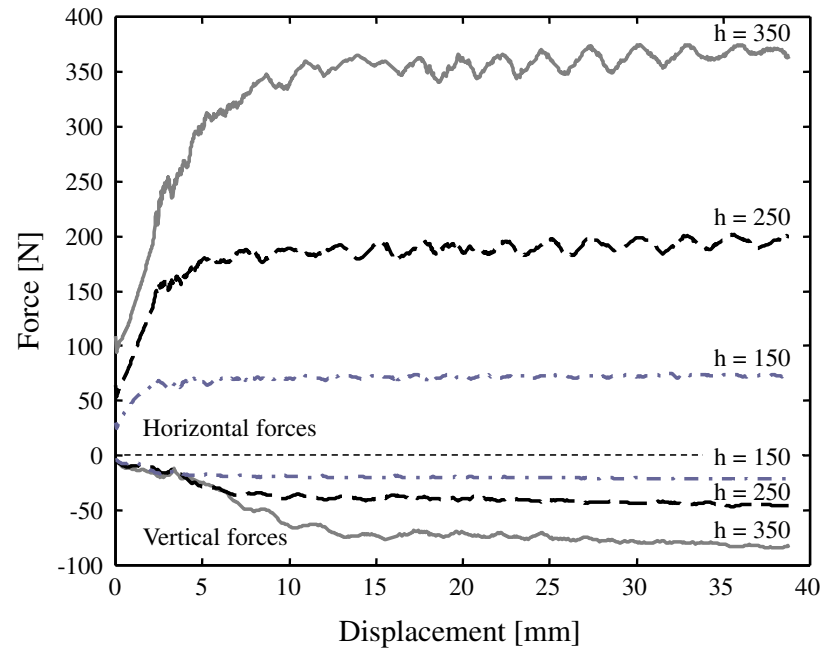

Fig. 7 The effect of blade depth $h$ on the blade forces using nonpolar MPM and $\psi=2^{\circ}$

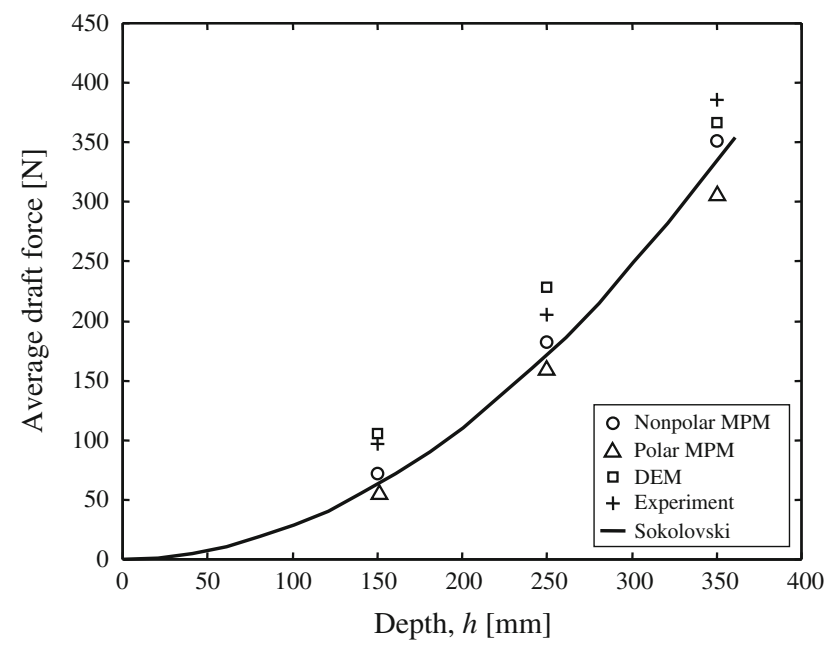

Fig. 8 Comparison between average draft forces: MPM, DEM, experiments and Sokolovski's method

mal stress, but the shear stress with free rotation, is almost half the stress when no rotation is allowed. This means that the rotation has an influence on the friction between the material and the blade. This has also been observed by Tejchman [24].

Taking the DEM average contacting force of two neighbouring contacts and dividing it by the distance between the contacting points along the blade, the stress at the blade could be estimated. Due to the particles moving in a step-like fashion, a time average was used to calculate the stress at the blade. The average was calculated between a blade displacement of 18 and $22 \mathrm{~mm}$ and the result is shown in Fig. 10. The normal and shear stress are lower than the stresses predicted by Sokolovski's method, but higher than the nonpolar MPM stresses.

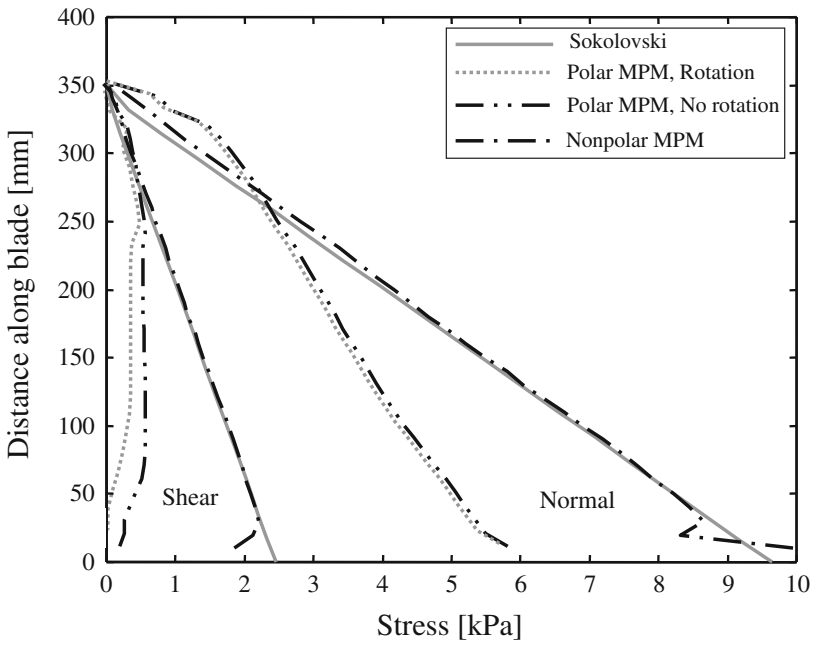

Fig. 9 Normal and shear stress at the blade for $h=350 \mathrm{~mm}$ : Nonpolar MPM, polar MPM and Sokolovski's method. Blade displacement $=$ $20 \mathrm{~mm}$

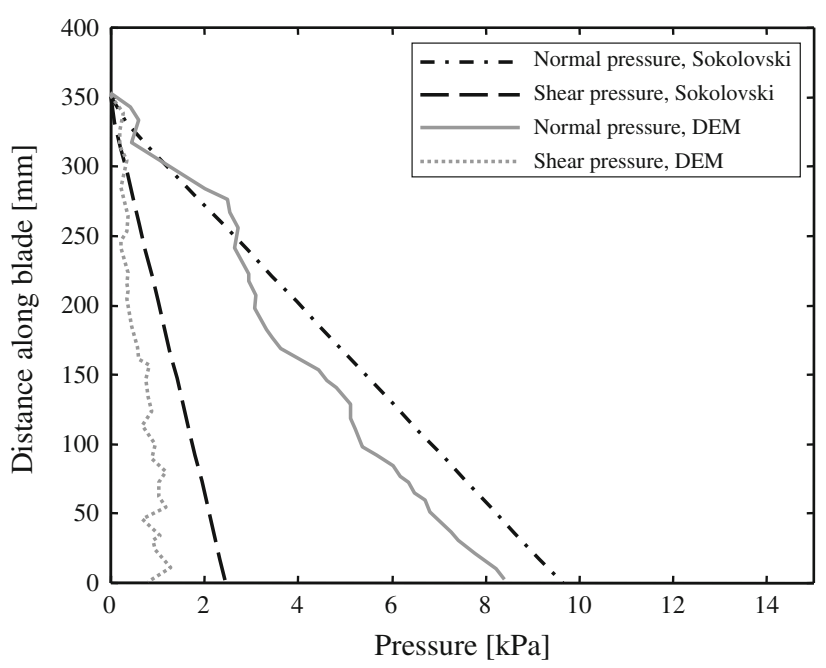

Fig. 10 Normal and shear stress at the blade for $h=350 \mathrm{~mm}$ : DEM and Sokolovski's method

\subsection{Shear banding}

In the third part of the analysis, the ability of the different numerical methods to predict shear banding is investigated. Again, only blade displacements up to $35 \mathrm{~mm}$ are considered, i.e., the effect of upheaving of material is avoided.

When the blade pushes the material forward, a shear band develops, reaching from the bottom tip of the blade to the free surface. Using DEM, it can be very difficult to predict the shear bands, but there is definitely evidence that such bands exist. Making use of the particle displacement ratio (PDR), slip lines can be identified. PDR is defined as the ratio of the magnitude of the particle displacement vector to the magnitude of the blade displacement vector. Note that when the term slip line is used, it is assumed to be a slip plane 

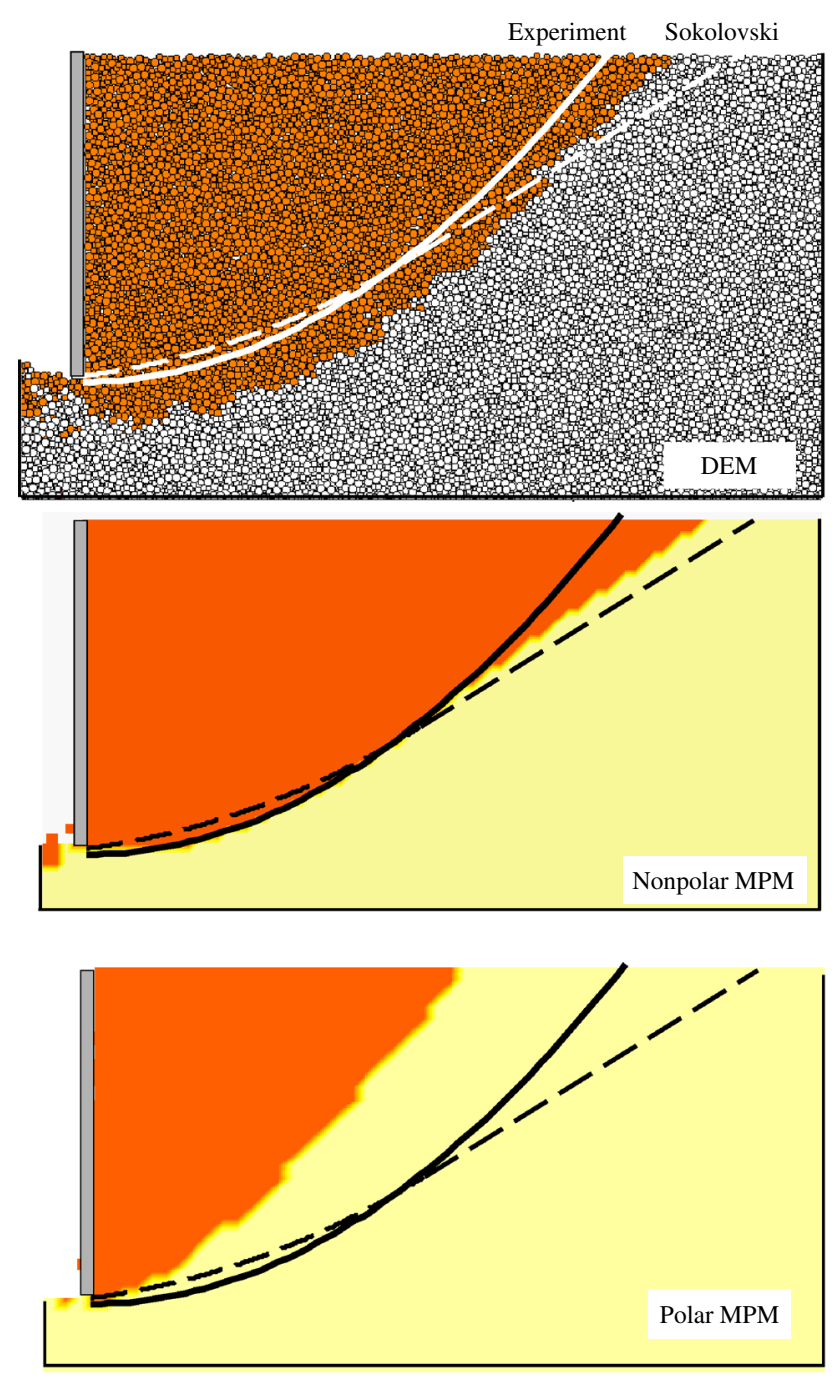

Fig. 11 Predicted slip lines using a particle displacement ratio $P D R=$ 0.15 . Blade displacement $=15 \mathrm{~mm}$ (DEM results from [20])

with no width, while a shear band is defined as a region of slip or a slip plane with a finite width. The top of Fig. 11 [20] shows the results using PDR $=0.15$, i.e., all the darker particles have been displaced a distance of at least 0.15 times the displacement of the blade). The experimentally observed slip line and the line predicted by Sokolovski's method are also shown. The line shown on the figure indicates the centre of the observed shear band. With PDR $=0.15$, the predicted slip lines falls between the observed line and the Sokolovski line at the free surface. Closer to the bottom edge of the blade, however, the slip line is outside of that predicted by Sokolovski's method. This is because the particles have a finite size whereas in Sokolovski's method, the slip line is assumed to start at the bottom edge of the blade.

The lower two images in Fig. 11 show similar results from nonpolar MPM and polar MPM respectively. All the material points within the darker region have PDR $\geq 0.15$. The nonpo-

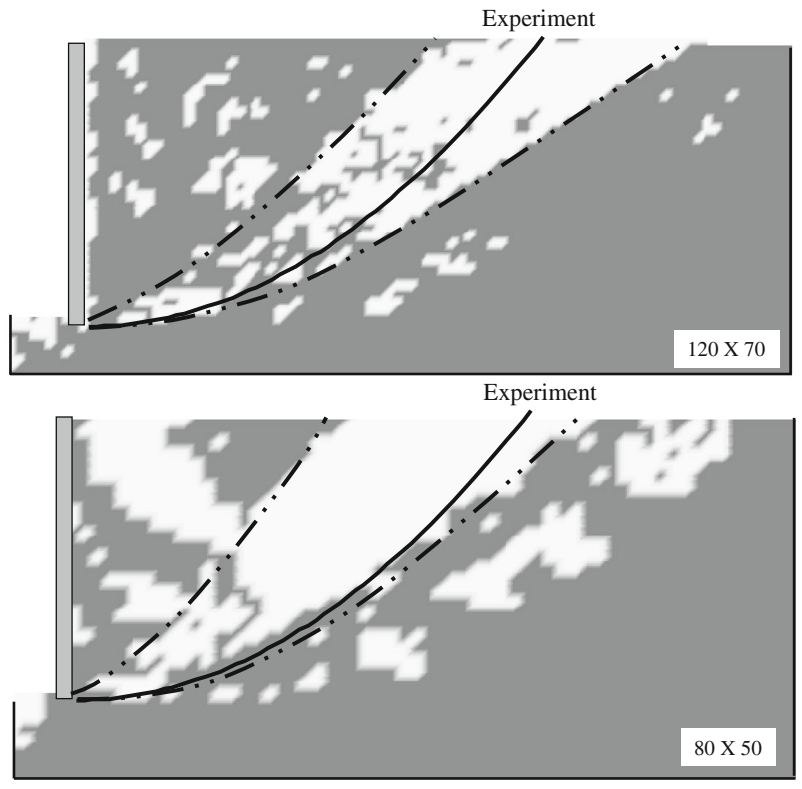

Fig. 12 Nonpolar MPM yield points: mesh sizes $120 \times 70$ and $80 \times 50$. Blade displacement $=15 \mathrm{~mm}$

lar MPM predicted slip line meets the free surface at almost exactly the same place as the DEM predicted slip line. Closer to the blade tip, however, the shear band more closely follows that of the observed and Sokolovski lines. Contrary to DEM modelling, in continuum modelling there are no particles with a finite size, which causes the DEM slip line to be outside the Sokolovski line. Polar MPM predicts a slip line at almost $45^{\circ}$ and it is less curved than the observed and nonpolar MPM slip lines.

The predicted slip lines can be manipulated by changing the PDR value to obtain a better fit. A more precise method of predicting the shear band is possible with a continuum method, but not with DEM. Using nonpolar MPM, Fig. 12 shows all the regions which have a stress state on the yield surface as lighter patches and those which have stress states below the yield surface (elastic state) as darker patches. Two mesh sizes were used, $120 \times 70$ and $80 \times 50$. Yield points are concentrated around the observed slip lines. The coarser mesh shows more yield points and a broader shear region as indicated by the dashed lines. Figure 13 shows the nonpolar MPM shear strain intensity $J^{\prime}=$ $\frac{1}{6}\left[\left(\varepsilon_{x x}-\varepsilon_{y y}\right)^{2}+\varepsilon_{y y}^{2}+\varepsilon_{x x}^{2}\right]+\varepsilon_{x y}^{2}$ with lighter regions indicating higher shear strains and vice versa. The shear strain shows a definite band, bounding the observed and Sokolovski slip lines. Again, mesh refinement shows convergence of the shear band width.

It is well-known that the Cosserat rotation is an indication of shear bands [24]. Figure 14 shows the rotation for two different mesh sizes with the rotation at the blade interface unconstrained (free). The shear zones can be identified by 

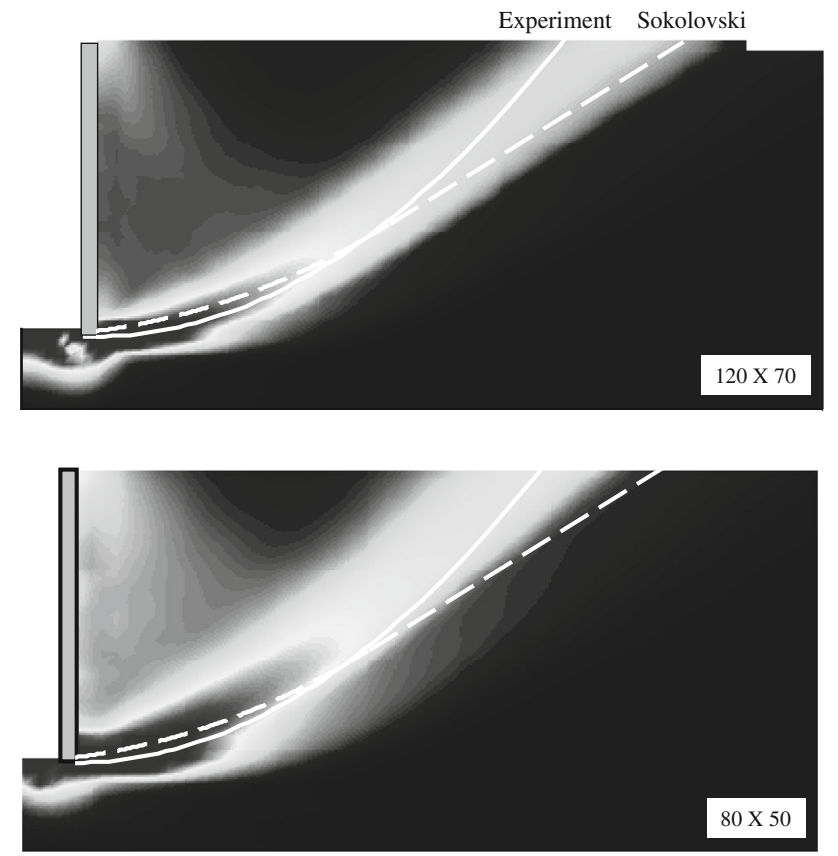

Fig. 13 Nonpolar MPM shear strains: mesh sizes $120 \times 70$ and $80 \times 50$. Blade displacement $=15 \mathrm{~mm}$

regions of high (negative) rotations. Again, the shear regions differ from the nonpolar MPM and experimental results, but do agree with the polar MPM displacement field as depicted in the bottom image of Fig. 11. There is little difference in the results from the two different mesh sizes. A mesh rotated through $35^{\circ}$ revealed that the orientation of the nonpolar MPM shear band is slightly influenced by the mesh orientation. The polar MPM shear band, however, showed to be independent of the mesh orientation.

The observed shear band had a width of about $7-8$ particle diameters, i.e., $70-80 \mathrm{~mm}$ [18]. The simulations, polar and nonpolar MPM, predict shear bands $60-80 \mathrm{~mm}$ wide. Tejchman [24] states that if the size of the elements is smaller than five times the mean grain diameter $d_{50}$, i.e. $5 \times 10 \mathrm{~mm}=50 \mathrm{~mm}$, the polar results are independent on the mesh size. The $120 \times 70$ and $80 \times 50$ mesh had element sizes $10 \mathrm{~mm} \times 10 \mathrm{~mm}$ and $15 \mathrm{~mm} \times 14 \mathrm{~mm}$ respectively. These sizes are well below five times the mean grain diameter.

The standard set of Cosserat constitutive coefficients $a_{1}=$ $0.375, a_{2}=0.125$ and $a_{3}=1$ was used. Muhlhaus and Vardoulakis [29] derived the values of these coefficients by taking into account slip and rotation in a random assembly of circular rods. Tejchman [24] investigated the effect of the Cosserat material parameters on biaxial simulations and concludes that the standard set of parameters turned out to be useful and sufficient in numerical calculations involving localisation. The influence of $a_{3}$ on the results was rather insignificant and, in general, the larger the difference between $a_{1}$ and $a_{2}$, the larger the non-symmetry of the stress tensor. Tejch-
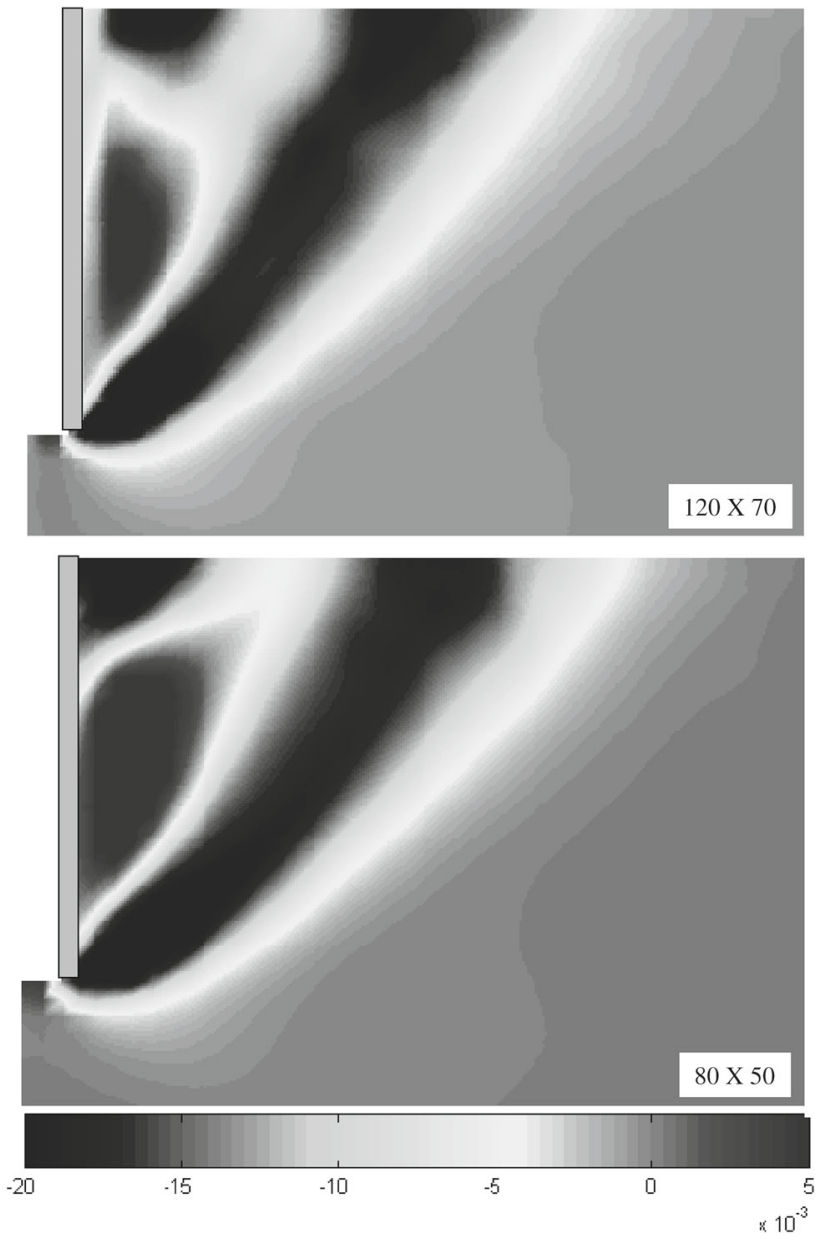

Fig. 14 Polar rotations [rad]: mesh sizes $120 \times 70$ and $80 \times 50$. Blade displacement $=15 \mathrm{~mm}$

man [24] also showed that when the following set is used, $a_{1}=0.25, a_{2}=0.25, a_{3}=0.5$, the response is slightly less stiff compared to the standard set. It was found in this study, that by changing the value of $G^{c}$ from $G^{c}=2 G$ to $G^{c}=0.5 G$ the draft force on the blade decreased by $1.3 \%$. Changing the internal length from $l=10 \mathrm{~mm}$ to $l=2 \mathrm{~mm}$, the draft force decreased by only $1.9 \%$. These results show that the model is not sensitive to changes in the values of $G^{c}$ and $l$.

\section{Conclusion}

DEM and MPM (nonpolar and polar) simulations were compared to experiments of a flat blade cutting through a cohesionless granular material.

Different dilatancy angles were used for nonpolar MPM modelling and it was found that an angle of $\psi=2^{\circ}$ resulted in flow patterns and free surface flows that closely resembled the observed behaviour. Using $\psi=2^{\circ}$, polar MPM could not 
accurately predict the free surface flow. Polar MPM showed less dilation, but compared well to the DEM results up to a displacement of $300 \mathrm{~mm}$. The dilatancy angle had a small influence on the draft force: an increase in dilatancy angle resulted in an increase in force. Nonpolar MPM predicted draft forces 9-25\% lower than the measured values for different blade depths. Polar MPM predicted the draft forces 20-30\% lower than the measured and 10- 28\% lower than the nonpolar MPM forces. For both nonpolar and polar MPM, the predicted draft forces were more accurate with an increase in blade depth, i.e. an increase in average stress. DEM predicted draft forces of $6 \%$ lower to $12 \%$ higher than the measured values.

Using a particle displacement ratio PDR $=0.15$, both nonpolar MPM and DEM could accurately predict the position and orientation of the observed shear band. Polar MPM predicted a straight shear band at $\pm 45^{\circ}$. Using the nonpolar shear strain and the polar rotations, more objective predictions of the shear bands could be done. The nonpolar approach showed to be more accurate in terms of predicting the position and orientation of the shear band, but also showed that the band width is mesh size dependent with a decrease in band width associated with mesh refinement. Although the polar approach showed that the mesh size had no significant effect on the shear band width, it failed to accurately predict the orientation of the shear band.

Using Sokolovski's method as reference, nonpolar MPM predicted normal and shear forces against the blade very accurately. Polar MPM failed to accurately predict the stresses and showed that the polar rotation at the blade interface had only a small influence on the stresses. DEM, in general, predicted lower stresses compared to Sokolovski's method, but higher than polar MPM.

Finally it is concluded that DEM and MPM can successfully model a flat blade cutting through a granular medium. This process is difficult to model with conventional FEM due to large displacements and severe distortion of the mesh. A standard set of Cosserat constitutive parameters was used together with the measured friction angle, dilation angle, Young's modulus and characteristic length. These additional parameters make it difficult to use the Cosserat continuum, unless accurate laboratory experiments are conducted to determine these parameters. In general, the Cosserat continuum performed worse than the nonpolar continuum. The effect of the different Cosserat parameters on soil flow, shear band orientation and width and blade-soil interface stresses needs further study. It is, however, possible if the Cosserat parameters can be determined more accurately that it will perform as good as the nonpolar continuum. The inherent complexity of the Cosserat continuum, however, makes it unsuitable for everyday practical use.

A cohesionless material was used in this study. The next logical step would be to include cohesion. Cohesion can be added to a DEM model in the form of inter-particle bonds
[30]. The strength and stiffness of these bonds can be based on the capillary and dynamic viscous forces induced by the presence of water between soil particles. Cohesion can easily be added to the MPM models since it is already included in the constitutive models used, but set to zero in this study.

This study also focused on two-dimensional modelling. In the case of a long flat blade and other limited cases this assumption is valid. When the flow is more complex around a three-dimensional tool, the numerical models have to be extended to capture the three-dimensional effects. Both DEM and MPM can be used to model tools of more complex geometry. Three-dimensional modelling (continuum and discrete) will be computationally more expensive than two-dimensional modelling and the cost will be determined by the size of the domain modelled, the number of elements (continuum) and the number of particles (discrete). Three-dimensional DEM codes are available [22] and MPM has recently been extended to three-dimensions [37].

Computation time using DEM and MPM was found to be comparable. Polar MPM was found to be $\pm 50 \%$ slower than nonpolar MPM. The DEM model used was fairly small, and it is expected that with an increase in the number of particles in the DEM model, computation times would increase and MPM would be the preferred method of choice.

\section{Appendix: The material-point method}

The basic formulation of MPM is presented here. For a more detailed description, see [19] and one of the pioneering papers by Sulsky and Schreyer [13]. In the first part of this appendix, index notation is used to denote vectors and matrices. The subscripts $i, j$ and $k$ are used, assuming values of 1 , 2 and 3, unless stated otherwise. In the latter part of the appendix, Hassenpflug [31] notation is used. A column vector is indicated by a overbar, $\overline{\boldsymbol{x}}$, a row vector by a underbar, $\underline{\boldsymbol{x}}$ and a matrix by both, $\underline{\overline{\boldsymbol{x}}}$. Plane strain conditions are assumed.

\subsection{Space discretisation}

First, the initial configuration of the body is divided into a number of subregions. This is done as depicted in Fig. 15. In the centre of each subregion a material point or particle is placed. This material point represents the subspace, and is given a mass $m_{p}$. The mass is calculated by assuming that the whole mass of the subregion is concentrated at the material point. The mass of a material point is constant and does not change with time or position. The density $\rho\left(x_{i}\right)$ represented by this collection of discrete mass points is approximated using the Dirac delta function 


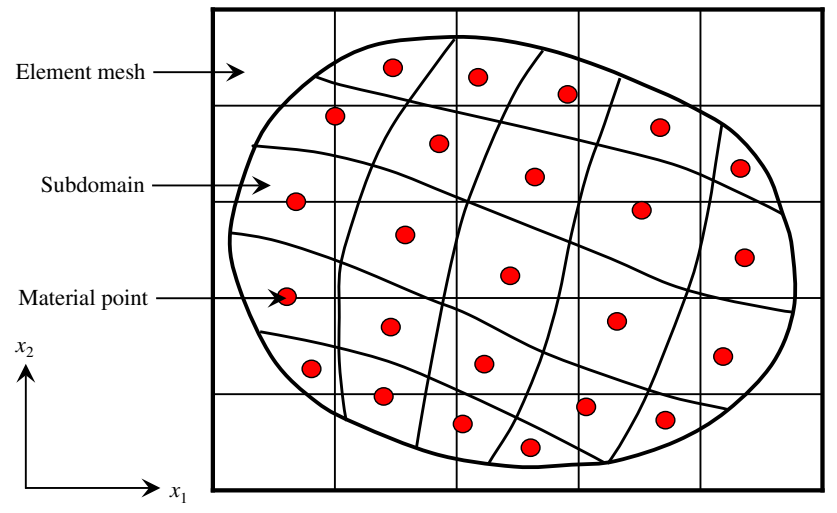

Fig. 15 Typical MPM discretisation indicating the finite elements and material points

$\rho\left(x_{i}\right)=\sum_{p=1}^{N_{p}} m_{p} \delta\left(x_{i}-x_{i}^{p}\right)$

where $x_{i}$ is an arbitrary position vector, $x_{i}^{p}$ is the position vector at material point $p, N_{p}$ is the total number of material points and the Dirac delta function is defined as follows

$\delta(x-a)=\left\{\begin{array}{ll}0 & x \neq a \\ \infty & x=a\end{array}\right.$ and $\int_{-\infty}^{+\infty} \delta(x-a) d x=1$

For clarity, the equations of motion are derived for a single element only. The whole system would be analysed by assembling the matrices and vectors as in standard FEM routines. The mass of each material point is fixed which ensures mass conservation.

The weak from of the linear momentum equation is given by

$$
\begin{aligned}
\int_{V} \rho \frac{d v_{i}}{d t} w_{i} d V= & \int_{V} \rho f_{i} w_{i} d V \\
& -\int_{V} \rho \sigma_{i j}^{S} w_{i, j} d V+\int_{S} \tau_{i} w_{i} d S
\end{aligned}
$$

where $v_{i}$ is the velocity vector, $f_{i}$ the body forces and $\tau_{i}$ the surface traction acting on surface $S$ and $w_{i}$ is test function. $\sigma_{i j}^{S}$ is the specific stress tensor defined as the Cauchy stress tensor divided by the material density. Substitution of the discrete density representation, Eq. (1), into Eq. (3) and making use of the definition of the Dirac delta function yields an discrete expression where the integration is performed as a sum of material point properties.

$$
\begin{aligned}
\sum_{p=1}^{N_{p}} m_{p} \frac{d v_{i}^{p}}{d t} w_{i}^{p}= & \sum_{p=1}^{N_{p}} m_{p} f_{i}^{p} w_{i} \\
& -\sum_{p=1}^{N_{p}} m_{p} \sigma_{i j}^{s p} w_{i, j}^{p}+\int_{S} \tau_{i} w_{i} d S
\end{aligned}
$$

The superscript $p$ indicates a variable evaluated at the material point. For example, the specific stress $\sigma_{i j}^{s p} \equiv \sigma_{i j}^{s p}\left(x_{i}^{p}\right)$.

\subsection{Element formulation}

The element mesh used is similar to that of FEM. Four noded quadrilateral elements are used. Under the assumption of two-dimensional conditions, the acceleration field $\dot{v}_{i} \equiv \frac{d v_{i}}{d t}$, for example, can be written in terms of nodal- and shape function-values

$\dot{\overline{\boldsymbol{v}}}(\overline{\boldsymbol{x}}, t) \equiv\left[\begin{array}{ll}\dot{v}_{1} & \dot{v}_{2}\end{array}\right]^{\mathrm{T}}=\underline{\overline{\boldsymbol{N}}} \dot{\boldsymbol{v}}^{n}$

where $\overline{\underline{N}}$ is a matrix containing the shape functions. The element nodal acceleration vector, $\dot{\bar{v}}^{n}$, contains the nodal values of the acceleration field. The same can be applied to the vector field $w_{i}$ to obtain a vector $\overline{\boldsymbol{w}}$. Define the following vectors under plane strain conditions,

$\overline{\boldsymbol{\sigma}}^{s} \equiv\left[\begin{array}{c}\sigma_{11}^{s} \\ \sigma_{22}^{s} \\ \sigma_{12}^{s}\end{array}\right], \quad \overline{\boldsymbol{f}} \equiv\left[\begin{array}{l}f_{1} \\ f_{2}\end{array}\right], \quad \overline{\boldsymbol{\tau}} \equiv\left[\begin{array}{c}\tau_{1} \\ \tau_{2}\end{array}\right]$

with $\sigma_{12}^{s}=\sigma_{21}^{s}$. Note that under the assumption of plane strain, the third normal stress component $\sigma_{33}^{s}$ is not included in the definition above. This component is, however, calculated at each material point and used in the constitutive model. Using these definitions, Eq. (4) can be written as follows

$$
\begin{gathered}
\overline{\boldsymbol{w}}^{\mathrm{T}} \sum_{p=1}^{N_{p}} m_{p}\left(\underline{\overline{\boldsymbol{N}}}^{p}\right)^{\mathrm{T}} \underline{\overline{\boldsymbol{N}}}^{p} \dot{\overline{\boldsymbol{v}}}^{n}=\overline{\boldsymbol{w}}^{\mathrm{T}} \sum_{p=1}^{N_{p}} m_{p}\left(\underline{\overline{\boldsymbol{N}}}^{p}\right)^{\mathrm{T}} \overline{\boldsymbol{f}}^{p} \\
-\overline{\boldsymbol{w}}^{\mathrm{T}} \sum_{p=1}^{N_{p}} m_{p}\left(\underline{\overline{\boldsymbol{B}}}^{p}\right)^{\mathrm{T}} \overline{\boldsymbol{\sigma}}^{s p}+\overline{\boldsymbol{w}}^{\mathrm{T}} \int_{S}\left(\underline{\overline{\boldsymbol{N}}}^{p}\right)^{\mathrm{T}} \overline{\boldsymbol{\tau}} d S
\end{gathered}
$$

where the superscript $p$ indicates values to be evaluated at the material points, e.g. $\underline{\bar{N}}^{p} \equiv \underline{\bar{N}}\left(\overline{\boldsymbol{x}}^{p}\right)$. The arbitrary test vector $\bar{w}$ appears in all the above terms and can thus be dropped. The final discretised system of equations follows as

$$
\underline{\overline{\boldsymbol{M}}} \dot{\dot{\boldsymbol{v}}}^{n}=\overline{\boldsymbol{F}}^{\mathrm{int}}+\overline{\boldsymbol{F}}^{\mathrm{ext}}
$$

where the mass matrix $\underline{\bar{M}}$ is given by

$M_{i j} \equiv \underline{\overline{\boldsymbol{M}}}=\sum_{p=1}^{N_{p}} m_{p}\left(\underline{\overline{\boldsymbol{N}}}^{p}\right)^{\mathrm{T}} \underline{\overline{\boldsymbol{N}}}^{p}$ 
the internal force vector is given by

$F_{i}^{\mathrm{int}} \equiv \overline{\boldsymbol{F}}^{\mathrm{int}}=-\sum_{p=1}^{N_{p}} m_{p}\left(\underline{\overline{\boldsymbol{B}}}^{p}\right)^{\mathrm{T}} \overline{\boldsymbol{\sigma}}^{s p}$

and the external force vector is given by

$F_{i}{ }^{\mathrm{ext}} \equiv \overline{\boldsymbol{F}}^{\mathrm{ext}}=\int_{S}\left(\underline{\overline{\boldsymbol{N}}}^{p}\right)^{\mathrm{T}} \overline{\boldsymbol{\tau}} d S$

The matrix $\underline{\boldsymbol{B}}^{p}$ contains shape function gradients. In practice, to simplify computations, a lumped mass matrix may be used instead of the consistent mass matrix given by Eq. (9). The lumped mass matrix is a diagonal matrix with each entry being the corresponding row sum of the consistent mass matrix. Matrix inversions become trivial if a lumped matrix is used, at the cost of introducing a small amount of numerical dissipation $[32,33]$. The consistent mass matrix can also be singular for certain arrangements of the particles. There appears to be only a few arrangements of particles that yield a singular consistent mass matrix, but nearby arrangements might result in an ill-conditioned matrix. On the other hand, the lumped matrix is diagonal and well conditioned.

\subsection{Time integration}

Let the time step size be $\Delta t$. The solution to the system of Eq. (8) is found at discrete instants in time $t, t+1, \ldots, t+$ $n$. The calculation during each time increment consists of three phases; a initialisation phase, a Langrangian phase and a convective phase [34].

\subsubsection{Initialisation phase}

Assume that the position and velocity vector, stress tensor, strain tensor and history dependent variables of each material point are known at time $t$. With the position of each particle known, its shape function values can be computed and hence the mass matrix, $\underline{\bar{M}}^{t}$, given by Eq. (9). Mapping of the particle velocities to the nodes, provides the initial data for the solution to Eq. (8). The following equation is solved to obtain the nodal velocity $\overline{\boldsymbol{v}}^{n, t}$ at time $t$.

$\underline{\overline{\boldsymbol{M}}}^{t} \overline{\boldsymbol{v}}^{n, t}=\sum_{p=1}^{N_{p}} m_{p}\left(\underline{\overline{\boldsymbol{N}}}^{p, t}\right)^{\mathrm{T}}\left[\begin{array}{l}v_{1}^{p, t} \\ v_{2}^{p, t}\end{array}\right]$

where $\left[v_{1}^{p, t} v_{2}^{p, t}\right]^{\mathrm{T}}$ is the material point velocity vector at time $t$, containing the velocity components in the $x_{1}$ - and $x_{2}$ directions respectively. This equation expresses equivalence of momentum calculated for the material points and for the nodes [35].

\subsubsection{Lagrangian phase}

With the shape functions of each particle known, the internaland external-force vectors can be calculated using Eqs. (10) and (11) respectively. With these two vectors and the mass matrix known, Eq. (8) is solved for the nodal acceleration at time $t$.

$\dot{\overline{\boldsymbol{v}}}^{n, t}=\left(\underline{\overline{\boldsymbol{M}}}^{t}\right)^{-1}\left(\overline{\boldsymbol{F}}^{\mathrm{int}, t}+\overline{\boldsymbol{F}}^{\mathrm{ext}, t}\right)$

The "new" nodal velocity, $\overline{\boldsymbol{v}}^{n, t+1}$, at time $t+1$ is obtained by using an explicit time integrator

$\overline{\boldsymbol{v}}^{n, t+1}=\overline{\boldsymbol{v}}^{n, t}+\Delta t \dot{\boldsymbol{v}}^{n, t}$

which obviously requires very small time steps to ensure accuracy. Using the new nodal velocity, the increment in strains can be calculated at the particles. Define the vector of strain increment as follows

$\Delta \overline{\boldsymbol{\varepsilon}}^{p} \equiv\left[\begin{array}{lll}\varepsilon_{11}^{p} & \varepsilon_{22}^{p} & \varepsilon_{12}^{p}\end{array}\right]^{\mathrm{T}}$

This vector can be calculated using matrix $\underline{\overline{\boldsymbol{B}}}^{p, t}$

$\Delta \overline{\boldsymbol{\varepsilon}}^{p, t+1}=\Delta t \underline{\overline{\boldsymbol{B}}}^{p, t} \overline{\boldsymbol{v}}^{n, t+1}$

With the increment in strain known, the new stress state $\overline{\boldsymbol{\sigma}}^{p, t+1}$ at each material point can be calculated based on the chosen constitutive model. One way of doing this, would be to calculate the stress increment, using the tangent modulus $\underline{\overline{\boldsymbol{M}}}^{p, t}$

$\Delta \overline{\boldsymbol{\sigma}}^{p, t+1}=\underline{\overline{\boldsymbol{M}}}^{p, t} \Delta \overline{\boldsymbol{\varepsilon}}^{p, t+1}$

The use of the tangent modulus in a numerical algorithm, however, results in a tendency for the stress to drift from the yield surface [13]. In practice a incremental iterative scheme is rather used. With the given increment in strain, the material is assumed to be elastic, and a trial stress state is computed. The yield function $f$ is evaluated using the trial state, and if $f \leq 0$ the material point is still in the elastic region and no further calculations are needed. However, if $f>0$, return algorithms, based on the flow potential $g$, are needed to force $f$ back to zero. In the case of a Mohr-Coulomb or DruckerPrager model, a simple one-step return to the yield surface is possible [23]. For other models like Lade, a iterative procedure is needed.

History dependent variables such as strain-hardening parameters may also be updated at this stage. During the Lagrangian phase the nodes are assumed to move at the computed nodal velocity $\overline{\boldsymbol{v}}^{n, t+1}$. Thus, points in the interior of the element move in proportion to the motion of the nodes, as 
given by the representation using the nodal shape functions. Since shape functions are used to map the nodal velocity continuously to the interior of the element, the positions of the material points are updated by moving them in a singlevalued, continuous velocity field. Similarly, the velocity of a material point is updated by mapping the nodal accelerations to the material point position. The updated position vector and velocity vector follow as

$$
\begin{aligned}
& \overline{\boldsymbol{x}}^{p, t+1}=\overline{\boldsymbol{x}}^{p, t}+\Delta t \underline{\overline{\boldsymbol{N}}}^{p, t} \overline{\boldsymbol{v}}^{n, t+1} ; \\
& \overline{\boldsymbol{v}}^{p, t+1}=\overline{\boldsymbol{v}}^{p, t}+\Delta t \underline{\overline{\boldsymbol{N}}}^{p, t} \dot{\boldsymbol{v}}^{n, t}
\end{aligned}
$$

Because the velocity field is single-valued, interpenetration of material is precluded. This feature of the algorithm allows simulations of impact and penetration without the need for a special contact algorithm.

\subsubsection{Convective phase}

At this point in the computational cycle, the material points are completely updated and carry the complete solution, i.e., all the state variables needed to start a new calculation step are carried by the material points. During the convective phase, the material points are held fixed and the element mesh can be redefined. The mesh can be chosen in any convenient manner, for example adaptive meshes can be used to resolve sharp gradients and interfaces. The simplest and most convenient choice is, however, to keep the existing mesh. Any motion of the mesh relative to the material points model convection. Since the material points do not move during the convective phase, material point properties have the same value at the end of the convective phase as they had at the end of the Lagrangian phase. This completes the computational cycle. A new cycle is begun using the information carried by the material points to initialise nodal values on the element mesh.

\subsection{Stability}

In the previous sections, a simple explicit time integrator is used of which the time step should satisfy the stability condition, i.e. the critical time step should be the smallest ratio of the element size to the wave speed through the material. For small displacements, the spatial discretisation in MPM is equivalent to that of FEM using Gauss points at the same locations as those of the material points in each element. Therefore, the convergence behaviour of the integrator used is similar to that employed to integrate the corresponding equations in FEM. However, according to Chen et al. [36], no consistent theoretical results have been obtained for the convergence behaviour of time integrators when larger deformations occur and a reasonable time step is usually found through numerical experiments.

\subsection{Contact model}

The MPM formulation presented here automatically enforces no-slip contact between two different bodies. The reason for this is that a single-valued velocity field is used for updating the particle positions. Bardenhagen et al. [14], however, developed an algorithm which relaxes the no-slip condition and allows Coulomb friction and slip at contacting boundary nodes. This contact algorithm was implemented to model the blade-soil interface. The friction angle between the corn and the steel blade is given in Table 1 . The theory behind the contact model is not presented here and the reader is referred to Bardenhagen et al. [14].

\section{References}

1. Karmakar S, Kushwaha R (2005) Dynamic modeling of soil-tool interaction: an overview from a fluid flow perspective. J Terramech (in press)

2. Hansen JB (1961) Earth pressure calculation. The Danish Technical Press, Copenhagen

3. Perumpral JV, Chance LC, Woeste FE, Desai CS (1980) A matrix method for force and moment analysis on a tillage tool. Trans. ASAE 1072

4. Perumpral JV, Grisso RD, Desai CS (1983) A soil-tool model based on limit equilibrium analysis. Trans. ASAE 991

5. Sokolovski VV (1954) Statics of soil media. Butterworth Scientific Publishers, London

6. Cundall PA, Strack ODL (1979) A discrete numerical method for granular assemblies. Geotechnique 29:47-65

7. Franco Y, Rubinstein D, Shmulevich I (2005) Determination of discrete element model parameters for soil-bulldozer blade interaction. In: Proceedings of the 15th international conference of the ISTVS. Hayama, Japan

8. Asaf Z, Rubinstein D, Shmulevich I (2005) Determination of discrete element model parameters using in-situ tests and inverse solution techniques. In: Proceedings of the 15th international conference of the ISTVS. Hayama, Japan

9. Bohatier C, Nouguier C (2000) Dynamic soil-tool interaction forces and flow state. In: International conference on applied mechanics. Durban, South Africa, pp 294-299

10. Bohatier C, Nouguier C (1999) Numerical modelling of large deformations in soils: continuum or granular medium. Energy sources technology conference \& exhibition, ETCE99-6713, ASME

11. Wieckowski $Z$ (2002) The dynamic analysis of large strain problems by the material point method. In: Fifth world congress on computational mechanics, WCCM V. TU Vienna, Vienna, Austria. Online at http://wccm.tuwien.ac.at

12. Benson DJ (1992) Computational methods in Lagrangian and Eulerian hydrocodes. Comput Methods Appl Mech Eng 99:235394

13. Sulsky D, Zhou SJ, Schreyer HL (1995) Application of a particlein-cell method to solid mechanics. Comput Phys Commun 87:236252

14. Bardenhagen SG, Brackbill JU, Sulsky DL (2000) The materialpoint method for granular materials. Comput Methods Appl Mech Eng 187:529-541

15. Herrmann H (1999) Institute for computer applications: physics on supercomputers. University of Stuttgart, ICA1 Annual Report

16. Eringen CA (1999) Microcontinuum field theories, I: foundations and solids. Springer, New York 
17. de Borst R (1991) Simulation of strain localization: a reappraisal of the Cosserat continuum. Eng. Comput. 8:317-332

18. Coetzee CJ (2000) Forced granular flow. Msc Thesis Mechanical Engineering, University of Stellenbosch, Stellenbosch, South Africa

19. Coetzee CJ (2004) The modelling of granular flow using the particle-in-cell method. Phd Thesis Mechanical Engineering, University of Stellenbosch, Stellenbosch, South Africa

20. Coetzee CJ, Els DNJ (2009) Calibration of granular material parameters for DEM modelling and numerical verification by bladegranular material interaction. J Terramech 46:15-26

21. Rowlands JC (1991) Dragline bucket filling. Phd Thesis, University of Queensland, Queensland, Australia

22. Itasca (1998) PFC2D: User Manual, Theory and Background, Version 2.0., www.itascacg.com

23. FLAC (1998) Fast lagrangian analysis of continua: theory and background. www.itascacg.com

24. Tejchman J (1997) Modelling of shear localisation and autogeneous dynamic effects in granular bodies. des Institutes fur Bodenmechanik und Felsmechanik der Universitat Fridericiana in Karlsruhe

25. Mouazen AM, Neményi M (1998) A review of the finite element modelling techniques of soil tillage. Math Comput Simul 48:23-32

26. Maciejewski J, Jarzebowski A, Trampczynski W (2004) Study on the efficiency of the digging process using the model of excavator bucket. J Terramech 40:221-233

27. Vermeer PA, de Borst R (1984) Non-associated plasticity for soils, concrete and rock. Heron 29(3):317-332
28. Jaky J (1944) The coefficient of earth pressure at rest. J Soc Hung Archit Eng, 355-358

29. Muhlhaus H-B, Vardoulakis I (1987) The thickness of shear bands in granular materials. Geotechnique 37:271-283

30. Zhang R, Jianqiao L (2005) Simulation on mechanical behaviour of cohesive soil by distinct element method. J Terramech (in press)

31. Hassenpflug WC (1993) Matrix tensor notation Part I: rectilinear orthogonal coordinates. Comput Math Appl 26(3):55-93

32. Burgess D, Sulsky D, Brackbill JU (1992) Mass matrix formulation of the FLIP particle-in-cell method. J Comput Phys 103:1-15

33. Brackbill JU, Kothe DB, Ruppel HM (1988) A low dissipation particle-in-cell method for fluid flow. Comput Phys Commun 48:25-38

34. Sulsky D, Chen Z, Schreyer H (1994) A particle method for historydependent materials. Comput Methods Appl Mech Eng 118:179196

35. Wieckowski Z (2001) Analysis of granular flow by the material point method. European Conference on Computational Mechanics. Cracow, Poland

36. Chen Z, Hu W, Shen L, Xin X, Brannon R (2002) An evaluation of the MPM for simulating dynamic failure with damage diffusion. Eng Fract Mech 69:1873-1890

37. Jassim I, Coetzee C, Vermeer PA (2013) A dynamic material point method for geomechanics. In: Hicks et al (eds) Installation Effects in Geotechnical Engineering. Taylor \& Francis Group, London 